\title{
La empresa social: contexto mexicano
}

\section{The Social Enterprise: Mexican Context}

\author{
Cristian Alejandro Rubalcava de León \\ Universidad Autónoma de Tamaulipas, México \\ cristianrl11933@hotmail.com \\ (D) http://orcid.org/0000-0002-9926-2902 \\ Mariana Zerón Félix \\ Universidad Autónoma de Tamaulipas, México \\ zfmariana@docentes.uat.edu.mx \\ (1) http://orcid.org/0000-0003-0584-6884
}

Recepción: 25 de abril de 2019

Aprobación: 11 de junio de 2019

\section{RESUMEN}

Se busca determinar los factores del entorno que contribuyen a la existencia de empresas sociales en México. El estudio emplea información proveniente de diversas fuentes secundarias al recolectar diferentes indicadores sobre dicho país. Los datos son analizados a través del uso de la técnica de regresión logística y los resultados permiten corroborar un efecto positivo y significativo del acceso a la educación y la facilidad de emprender en la existencia de empresas sociales. En consecuencia, el estudio es un acercamiento al panorama que tiene México sobre el emprendimiento social y la empresa social.

Palabras clave: empresa social, emprendimiento social, acceso a la educación.

\begin{abstract}
The objective of this article is to determine the environmental factors that contribute to the existence of social enterprises in Mexico. The study makes use of information from various secondary sources collecting different indicators about that country. The data is analyzed through the use of the Logistic Regression techniques and the results allow us to corroborate a positive and significant effect of access to education and the ease of undertaking in the existence of social enterprises. Consequently, the study is an approach to the panorama that Mexico has about Social Entrepreneurship and Social Enterprise.
\end{abstract}

KEYWORDS: social enterprise, social entrepreneurship, access to education.

\section{INTRODUCCIÓN}

En la última década ha surgido un interés, por parte de investigadores, hacia la construcción de nuevos conocimientos sobre el emprendimiento social, el cual en la actualidad se conoce como un proceso que emplea técnicas administrativas con el objetivo de ofrecer bienestar y valor social hacia los sectores de la población que se encuentran en situaciones de vulnerabilidad (Mair y Martí, 2006; Urbano et al., 2014). Este proceso incluye la actuación coordinada de diferentes actores como el gobierno, el sector privado y la misma sociedad (Urbano et al., 2014). Dado lo anterior, el emprendimiento social es un elemento clave para lograr desarrollo, tanto económico como social, al poseer la ventaja de ofrecer mecanismos innovadores que faciliten la solvencia a problemáticas sociales como la pobreza, la discriminación o la exclusión, por citar algunas de ellas (Estrin et al., 2013; Urbano et al., 2010; Zahra et al., 2008).

No obstante, mientras que el emprendimiento comercial o tradicional ha ganado reconocimiento dentro de la literatura administrativa (Estrin et al., 2013), su contraparte social demanda la realización de nuevos estudios que lo fortalezcan (Kachlami et al., 2018; Urbano et al., 2017). Estos estudios deberán de ser índole cuantitativa, los cuales permitan amplificar el conocimiento sobre el emprendimiento social con la finalidad de alcanzar un esquema teórico que facilite la comprensión del tópico (Dwivedi y Weerawardena, 2018; Kachlami et al., 2018; Pathak y Muralidharan, 2018). 
La literatura sobre el emprendimiento social ha utilizado los efectos del entorno para evaluar el impacto que éste tiene en las diferentes aristas del proceso del emprendimiento social (Littlewood y Holt, 2018). Ejemplo de ello son los estudios que tienen como propósito evaluar el estudio de la intención de emprender socialmente (Pathak y Muralidharan, 2018), encontrar aquellos que favorecen la creación de empresas sociales (Hoogendoorn, 2016) o el enrolamiento de individuos en actividades sociales (Urbano et al., 2017), por mencionar algunos. Empero, no se encuentran estudios que relacionan el entorno y la existencia de empresas sociales.

Aunado a lo anterior, el emprendimiento social se ha relacionado con su contraparte comercial; esta situación ha desencadenado el uso de factores y metodologías que tienen sus bases y principios en el emprendimiento tradicional, los cuales son adaptados para ser operacionalizados en contextos de emprendimiento de tipo social (Austin et al., 2006; Felix Gonzalez et al., 2017). Este escenario complica la comprensión y claridad de los conceptos pertenecientes al campo de acción del emprendimiento social (Bacq y Janssen, 2011; Zahra et al., 2008).

Además, las investigaciones dirigidas al entorno se han concentrado exclusivamente en países con economías desarrolladas (Son et al., 2017; Urbano et al., 2017) sin tomar en cuenta a aquellos situados en una posición emergente (Littlewood y Holt, 2018). En Latinoamérica, se tienen ejemplos contados sobre el efecto del emprendimiento social y que, en su mayoría, resultan ser estudios de caso (Hlady-Rispal y Servantie, 2017). Bajo este panorama, la literatura en países con economías emergentes requiere desarrollarse a nuevos niveles, ya que se procura la obtención de nuevos conocimientos que permitan resaltar la importancia que tiene el tópico en la mejora de las condiciones de vida de las personas (Hlady-Rispal y Servantie, 2017; Wulleman y Hudon, 2016).

Particularmente, México resulta ser un país atractivo para la realización de estudios sobre la temática debido a que se tienen las condiciones para que sobresalgan actores que funden empresas sociales (Felix Gonzalez et al., 2017; Hlady-Rispal y Servantie, 2017; Saiz-Álvarez, 2018). Este tipo de organización se enmarca como un modelo de negocio que prioriza conseguir beneficios sociales en lugar de generar un retorno monetario; este último es usualmente empleado por dichas empresas para continuar con sus operaciones mientras procuran cumplir con la misión social por la cual fueron creadas (Roy et al., 2014; Son et al., 2017).

Sin embargo, la literatura que estudia el tópico en el país menciona que prevalece la necesidad de integrar una mayor cantidad de datos estadísticos sobre el proceso del emprendimiento social (Felix Gonzalez et al., 2017; Saiz-Álvarez, 2018); esta necesidad se genera a partir de la falta de estandarización de la noción sobre la empresa social mexicana (Fernández et al., 2012; Hlady-Rispal y Servantie, 2017) y a una alta complejidad para identificar a emprendedores sociales que se encuentran activos (Felix Gonzalez et al., 2017).

Contemplando el escenario anterior, se formula la pregunta de investigación: ¿Cuáles son los factores del entorno que determinan la existencia de empresas sociales en México? Para resolverla, se plantea como objetivo del estudio determinar los factores del entorno que contribuyen a la existencia de empresas sociales en México. De este modo, el artículo ofrece un acercamiento a la realidad del emprendimiento social en el escenario mexicano desde la perspectiva de la generación de empresas sociales, con lo cual se refuerza la literatura existente sobre el tema en países latinoamericanos (Felix Gonzalez et al., 2017; Felix-Gonzalez, 2011; Hlady-Rispal y Servantie, 2017; Saiz-Álvarez, 2018).

\section{REVISIÓN EMPÍRICA}

Se ha determinado que el emprendimiento social ha evolucionado en el transcurso del tiempo desde la propuesta inicial de Dennis R. Young en 1983 (Bacq y Janssen, 2011). Igualmente, se ha forjado a partir de comparaciones con el emprendimiento comercial, puesto que ambos tienen por finalidad mejorar las condiciones de vida de un país al incrementar sus niveles de bienestar económico y social (Austin et al., 2006; Estrin et al., 2013; Felix Gonzalez et al., 2017). La discrepancia más importante se da en la misión social que persigue cada uno de estos emprendedores (Austin et al., 2006) y su principal semejanza se da respecto a la forma en que crean y reconocen oportunidades para materializar futuros modelos de negocio (Bacq y Janssen, 2011; Felix Gonzalez et al., 2017). 
En el emprendimiento comercial es factible visualizar diferentes agrupaciones de diversos elementos y criterios sobre la creación de una nueva empresa, los cuales se aglomeran en cuatro conjuntos: $a$ ) las características del individuo, $b$ ) características de la organización, c) el proceso de emprendimiento y d) las condiciones del entorno (Gartner, 1985). En este último conjunto se encuentran las investigaciones que emplean factores económicos, sociales y culturales (Castaño et al., 2015), las cuales destacan la influencia de elementos propios de cada nación: a) la percepción del individuo sobre la corrupción (Aparicio et al., 2016), b) el rendimiento económico (Castaño et al., 2015), c) la productividad laboral (Aparicio et al., 2016), d) el producto interno bruto (Estrin et al., 2013), e) fuentes de financiamiento (Urbano et al., 2017),f) género (Aparicio et al., 2016; Estrin et al., 2013),g) inversión a investigación y desarrollo, así como a la apertura de mercados (inversión extranjera) (Castaño et al., 2015) y h) grado de estudio (Estrin et al., 2013), entre otras.

Estos estudios en su mayoría hacen uso de la teoría institucional (Aparicio et al., 2016; Castaño et al., 2015), la cual tiene su fundamento en los principios que cada país posee de las instituciones, por ejemplo las reglas y normas que tienen la capacidad de influenciar los procesos de desarrollo de la nación (North, 1991) para la formación y creación de nuevas empresas (Urbano et al., 2017).

A su vez, la teoría institucional asume que las instituciones se dividen en dos categorías. La primera, denominada formal, se refiere a los aspectos normativos y explícitos que rigen al ser humano; en este rubro se encuentran los contratos, procedimientos y criterios regulatorios. La segunda, designada como informal, es descrita con los fundamentos propios de cada sociedad como la cultura, los valores y las normas del tipo social (North, 1991; Urbano et al., 2017).

En este sentido, la teoría institucional ha sido abordada en el emprendimiento social de diferentes maneras cuando se busca investigar los efectos del entorno en esta temática (Hoogendoorn, 2016; Pathak y Muralidharan, 2018; Urbano et al., 2017,2014). Entre estos estudios se encuentran los que guardan relación con empresas sociales; referente a este modelo de negocio, se tiene evidencia relacionada con la intención del individuo de emprender socialmente (Pathak y Muralidharan, 2018; Urbano et al., 2017, 2010), a conocer la relevancia del entorno en este tipo de empresas que han comenzado a operar (Hoogendoorn, 2016; Littlewood y Holt, 2018) o si han desempeñado labores por periodos de más de tres años y siguen en funcionamiento (Kachlami et al., 2018).

Dentro de los criterios empleados para medir el impacto del entorno en el panorama social se encuentran rúbricas que son similares al comercial; es decir, se han utilizado índices de producto interno bruto (Kachlami et al., 2018; Urbano et al., 2017), la percepción de la corrupción, la productividad laboral (Kachlami et al., 2018), ingreso per cápita (Hoogendoorn, 2016), desigualdad económica (Pathak y Muralidharan, 2018), género (Urbano et al., 2014), grado de estudio (Kachlami et al., 2018; Saiz-Álvarez, 2018), por citar algunos.

No obstante, existen criterios únicos que se han empleado para evaluar los efectos del entorno en el emprendimiento social como los índices de altruismo, el posmaterialismo y ser partícipe de una organización social (Urbano et al., 2014), gasto público destinado al bienestar (Hoogendoorn, 2016), indicadores sobre rezago social como pobreza, acceso a la educación (Kachlami et al., 2018; Saiz-Álvarez, 2018), migración, comida, salud y servicios básicos (Saiz-Álvarez, 2018). La tabla 1 permite resumir las variables del entorno que se han empleado en el emprendimiento comercial y social.

TABLA 1

Indicadores y elementos del entorno

\begin{tabular}{|cll}
\hline Tipo de emprendimiento & \multicolumn{1}{c}{ Similares } & \multicolumn{1}{c}{ Diferentes } \\
\hline Comercial & Producto interno bruto, percepción sobre & $\begin{array}{l}\text { Gasto público a investigación y desarrollo, } \\
\text { apertura de mercados. }\end{array}$ \\
\cline { 3 - 4 } Social & $\begin{array}{l}\text { corrupción, productividad laboral, ingreso } \\
\text { per cápita, desigualdad económica, género }\end{array}$ & $\begin{array}{l}\text { Altruismo, gasto público a bienestar social, } \\
\text { migración, pobreza, acceso a comida, } \\
\text { educación, salud y servicios básicos en la } \\
\text { vivienda }\end{array}$ \\
\hline
\end{tabular}

Fuente: elaboración propia a partir de estudios sobre emprendimiento comercial y social (Aparicio et al., 2016; Castaño et al., 2015; Estrin et al., 2013; Hoogendoorn, 2016; Kachlami et al., 2018; Pathak y Muralidharan, 2018; Urbano et al., 2017, 2014). 
Las diferencias en los indicadores se deben principalmente a dos razones. La primera es la finalidad con la que se fundó el emprendimiento, es decir, si tuvo objetivos comerciales o sociales. La segunda se observa a partir de las características propias de cada individuo emprendedor, la cual se puede atribuir a sus rasgos de personalidad y cualidades referentes a su modo de pensar sobre las problemáticas que afectan al entorno que lo rodea (Austin et al., 2006; Braga et al., 2014). Estas diferenciaciones son las que determinan, en gran medida, el resultado que surgirá de un proceso emprendedor; es decir, la empresa social es producto del accionar y pensamiento de un sujeto con características que resguardan una afinidad social (Bacq y Janssen, 2011; Spear et al., 2009).

\section{Condición actual del proceso del emprendimiento social en México}

En relación con los efectos del entorno y el emprendimiento social, Littlewood y Holt (2018) sugieren que dicha asociación requiere seguir siendo explorada principalmente en países emergentes; para ello, realizan una evaluación sobre seis empresas sociales localizadas en el continente africano, específicamente en Sudáfrica, donde efectúan un análisis cualitativo que se modela a partir del esquema propuesto por Gartner (1985) con miras a ilustrar la importancia del entorno en la formación de cualquier tipo de emprendimiento.

Entre los resultados que ofrecen se encuentra la descripción de las principales problemáticas que afectan a Sudáfrica, desde el retrato de un contexto socioeconómico observado a través de la desigualdad económica, pobreza y crimen hasta las deficiencias de la parte institucional del país. En particular, estos autores, destacan la influencia positiva o negativa de este último elemento en el nacimiento de emprendedores y empresas sociales. Igualmente, mencionan que la empresa social sudafricana se ve limitada respecto a la cantidad de apoyo que recibe por fuentes externas; esta situación las obliga a volverse corporativas al mismo tiempo que dejan de lado los motivos iniciales por los que se crearon, así como por la nula conceptualización o nomenclatura de figura legal correcta que se adecue al funcionamiento de la empresa social. Esta aglomeración de problemas ocasiona que la empresa social tenga dificultades en su forma de operar (Littlewood y Holt, 2018).

Dicha situación, es muy similar a la de México (Felix Gonzalez et al., 2017; Felix-Gonzalez, 2011). En primera instancia, la amplia diversidad de características ambientales, culturales y sociales que predomina en los diferentes estados en los que se divide el territorio mexicano hace imposible homogeneizar los factores que favorecen efectuar acciones de emprendimiento (García-Macias et al., 2018). En segundo lugar, las dependencias gubernamentales $[1]$ encargadas de fomentar y apoyar las actividades de emprendimiento se encuentran particionadas, lo que provoca una nula coordinación para la formación de emprendedores del tipo social (Fernández et al., 2012; Inter-American Development Bank, 2016).

Además, es preciso mencionar que se tienen diferentes figuras normativas y jurídicas, pero ninguna de ellas es atribuible a englobar las características propias de este modelo de negocio (Inter-American Development Bank, 2016; Wulleman y Hudon, 2016); dicho de otro modo, se carece de un marco de trabajo regulatorio que permita clasificar una empresa como empresa social (Felix Gonzalez et al., 2017).

A pesar de lo anterior, la empresa social en México puede ser abordada a través de la definición que ofrece el Instituto Nacional de Desarrollo Social (INDESOL). Este organismo perteneciente al sector público se encarga de fortalecer los aspectos sociales del país y de registrar a organizaciones de la sociedad civil (OSC), a las cuales define de la siguiente manera:

Son agrupaciones constituidas por individuos, fundamentados en lazos asociativos que pueden realizar actividades de defensa y respeto a los derechos humanos, de apoyo o asistencia a terceros sin fines de lucro ni de proselitismo partidista, político-electoral o religioso, que no persiguen beneficios personales sino sociales comunitarios. Esencialmente, su origen responde al derecho de todo miembro de la sociedad de ejercer su participación ciudadana como la clave para la existencia de una mayor corresponsabilidad de los gobiernos y los ciudadanos (INDESOL, 2018a).[2] 
De la definición anterior se pueden extraer elementos que son propios de las conceptualizaciones sobre emprendimientos sociales clásicos (Dwivedi y Weerawardena, 2018; Wulleman y Hudon, 2016). Sin embargo, la definición que postula INDESOL es arbitraria, ya que realiza inclusiones sobre diferentes figuras jurídicas que poseen las OSC sin diferenciar si dicha figura hace omisión a la búsqueda de rendimientos financieros.

México, al igual que Sudáfrica, ha visto su desarrollo obstruido por la presencia de problemáticas que afectan a sus habitantes, pese a los esfuerzos del sector público por tratar de disminuirlas (Felix-Gonzalez, 2011; Saiz-Álvarez, 2018). De esta manera, el emprendimiento social es una herramienta esencial para reducir esos problemas problemas al demostrar su éxito en otros países (Hlady-Rispal y Servantie, 2017; Seelos y Mair, 2005). Con base en lo anterior, se plantea comprobar cuáles son los factores determinantes que generan la existencia de empresas sociales en el entorno mexicano.

\section{EMPRESA SOCIAL Y DESARROLLO HUMANO}

La detección de oportunidades para la creación de modelos de negocio del tipo social guarda una estrecha conexión con la presencia de problemáticas que afectan a las necesidades humanas; por ejemplo, la carencia en el suministro de medicamentos, alimentos y educación o las relacionadas con la inclusión social (Braga et al., 2014; Seelos y Mair, 2005). Bajo este contexto, una baja accesibilidad a niveles óptimos de bienestar y riqueza social, los cuales incluyen criterios del desarrollo humano como el aspecto económico, educativo y de salud, incrementarán la viabilidad de este modo de emprendimiento (Zahra et al., 2008).

Es así como el emprendimiento social se ha estudiado en conjunto con temas concernientes al rendimiento económico tanto de países como de personas (Kachlami et al., 2018; Levie y Hart, 2011; Pathak y Muralidharan, 2018; Urbano et al., 2017, 2014). Empleándola como variable de control, Urbano et al. (2017) expone que el emprendimiento social tiene una relación directa con el ingreso per cápita, la cual resulta ser negativa cuando es concerniente al género masculino.

Además, Pathak y Muralidharan (2018) demuestran que una alta desigualdad económica entre los habitantes de un país provocará que un mayor número de individuos se decanten por la creación de nuevas empresas sociales para solventar este problema. Desde otra perspectiva, Kachlami et al. (2018) proponen que las regiones que tengan un ingreso per cápita por encima del promedio serán idóneas para establecer empresas sociales. A consecuencia de los argumentos descritos, se propone la hipótesis siguiente:

$H_{1}$ : El bajo nivel de ingreso económico tiene un efecto positivo en la probabilidad de existencia de las empresas sociales en México.

Por su parte, la relación entre el emprendimiento social y la educación ha sido concebida ampliamente en la literatura (Estrin et al., 2013; Kachlami et al., 2018; Levie y Hart, 2011; Urbano et al., 2017). Referente a ello, Kachlami et al. (2018) afirman que la población que posee un alto nivel de preparación académica es idónea para la fundación de empresas sociales. Este resultado es similar al encontrado por Estrin et al. (2013), quienes sugieren que la educación desempeña un rol importante en las intenciones de emprender tanto comercial como socialmente. Además, indica que las personas con estándares educativos por encima del nivel profesional, es decir un posgrado, son más aptas para dirigir empresas sociales.

En contraposición, Urbano et al. (2017), al evaluar el contexto español, descubren que en ambos elementos, si bien se encuentran correlacionados, su significatividad no es relevante, por lo que asumen que altos niveles de formación educativa no tienen un impacto en las acciones en las que un emprendedor social se encuentre trabajando. Ante los argumentos descritos, se presenta la hipótesis correspondiente.

$\mathrm{H}_{2}$ : El alto acceso a la educación tiene un efecto positivo en la probabilidad de existencia de las empresas sociales en México. 
Por otro lado, el emprendimiento social y la salud se han relacionado mediante el estudio de organizaciones sociales que nacieron por el interés de entregar a la sociedad nuevas formas de cuidar la salud de las personas (Roy et al., 2014). De igual forma, en Reino Unido, Spear et al. (2009) indican que las empresas sociales con dicha área de acción han surgido por iniciativas del gobierno para su formación y desarrollo. Sin embargo, la sociedad de dicha nación prefiere a las organizaciones privadas que atienden la salud, a partir de que las consideran más innovadoras y responsivas que las que se encuentran operadas por el sector público (Roy et al., 2014).

El gobierno mexicano ha impulsado la creación de programas basados en el derecho universal a la salud que incrementen el alcance de prestaciones médicas de las personas; un ejemplo de esto es el Seguro Popular, que se caracteriza por atender a la población carente de accesos a servicios de salud, pero no ha logrado los resultados esperados (Felix-Gonzalez, 2011). Empero, Saiz-Álvarez (2018) menciona que los pueblos mexicanos donde se han detectado emprendimientos sociales han conseguido positivamente a los indicadores relacionados con la pobreza y salud. Ante esta situación, reluce la siguiente hipótesis:

$H_{3}$ : El bajo nivel de acceso a la salud tiene un efecto positivo en la probabilidad de existencia de las empresas sociales en México.

Ahora bien, Saiz-Álvarez (2018), para el caso de México, ilustra el hecho de que la presencia de los factores mencionados son causantes de un alto índice de migración; este último movimiento de población es considerado una de las razones principales por las que las empresas sociales pueden dejar de existir (Braga et al., 2014), pero aquellos sitios en donde se encuentren emprendimientos sociales han logrado reducir este indicador (Saiz-Álvarez, 2018). Contrario a estos puntos, Kachlami et al. (2018) revela que las empresas sociales tienden a nacer en lugares que se encuentran muy urbanizados a partir de que la sobrepoblación deriva en la creación de problemáticas. Por lo tanto, la hipótesis correspondiente es:

$H_{4}$ : El bajo nivel de migración tiene un efecto positivo en la probabilidad de existencia de las empresas sociales en México.

Igualmente, se plantea la utilización de variables relacionadas con la inseguridad, pues es un eje que representa una línea de acción, la cual requiere ser abordada por el emprendimiento social y ha sido poco explorada en la literatura (Littlewood y Holt, 2018). Como antecedente, se puede citar a Hoogendoorn (2016), quien encuentra que el hecho de contar con un entorno institucional fortalecido, con la aplicación correcta de leyes, deriva en que un ciudadano tenga la disposición necesaria para emprender socialmente al sentirse protegido del crimen.

Sin embargo, el paradigma donde se encuentra México es diferente debido a que sobresale por sus altos indicadores relacionados con acciones de crimen y violencia, lo cual se evidencia con una alta percepción negativa de sus habitantes sobre la seguridad, de donde se obtienen valores por encima de 70\% (Saiz-Álvarez, 2018). A pesar de ello, es factible localizar, en otros países, empresas sociales que operan en entornos teñidos por violencia (Hlady-Rispal y Servantie, 2017). En este aspecto, Pathak y Muralidharan (2018) sugieren que el emprendimiento social operará en primera instancia para reducir problemáticas, como la desigualdad económica, pero, en el largo plazo, representa un mecanismo que tiene la capacidad para lograr la disminución de indicadores afines al crimen. A consecuencia de ello se fundamenta la siguiente hipótesis:

$H_{5}$ : La alta percepción de inseguridad tiene un efecto positivo en la probabilidad de existencia de las empresas sociales en México.

En resumen, y fundamentando el hecho de que la creación de empresas sociales mediante el emprendimiento social significa una forma innovadora de crear nuevos modelos (Hlady-Rispal y Servantie, 2017; 
Seelos y Mair, 2005), se requieren evaluar aspectos relacionados con la facilidad de emprender que tendría un individuo que se desencante por este tipo de emprendimiento. Urbano et al. (2010) propone que en el momento cuando las instituciones formales reconozcan la importancia de este tipo de modelos, a través de la creación de apoyos que se produzcan en el sector público y privado, se fomentará el desarrollo de una mayor creación de éstos (Urbano et al., 2010; Wulleman y Hudon, 2016).

Lo anterior bajo el fundamento de que el acceso a un mayor financiamiento guarda una relación positiva con el nacimiento de emprendedores sociales, que es representado por mayores facilidades hacia la obtención de crédito bancario (Urbano et al., 2017), a lo que se debe agregar una flexibilidad en la creación de leyes que beneficien a las empresas sociales al incluir aspectos asociados con trámites legales y carga tributaria (Son et al., 2017). Por lo tanto, se redacta la siguiente hipótesis:

$H_{6}$ : La alta facilidad de emprender tiene un efecto positivo en la probabilidad de existencia de las empresas sociales en México.

A manera de resumen, se presenta el modelo correspondiente con las hipótesis propuestas para la investigación.

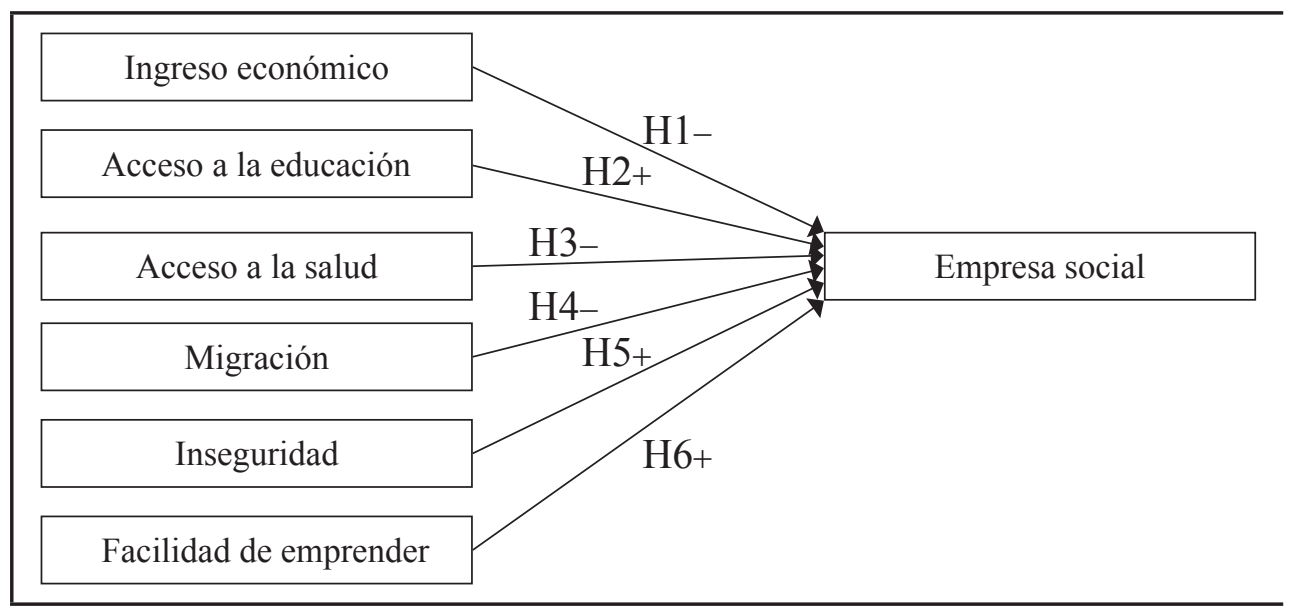

FIGURA 1

Modelo de factores del entorno y empresa social

Fuente: elaboración propia.

\section{Diseño de inVestigación}

\section{1. Unidad de análisis}

Para lograr el objetivo de la investigación, que consiste en determinar los factores del entorno que contribuyen en la existencia de empresas sociales en México, se utilizó como unidad de análisis a las 32 entidades federativas en las que se organiza el territorio mexicano. De esta manera, la información sobre el número de OSC se obtuvo a partir de aquellas que cuentan con un registro formalizado ante INDESOL (INDESOL, 2018b). De acuerdo con el registro, la población de este tipo de organización asciende a 37181 entidades distribuidas por el territorio de México. En el registro se incluye información sobre cada una de las OSC y se destacada el estatus en el que se encuentre a la fecha (activa o inactiva). Estas organizaciones están obligadas a actualizar sus registros una vez cada año; de no hacerlo en dos consecutivos, pasará a ser inactiva y se limitará su posibilidad de ser partícipe en convocatorias del sector público. 


\section{2. Definición de variable dependiente}

Siguiendo los lineamientos sobre el modelo tradicional LOGIT, se establece que el resultado de la variable dependiente es de tipo dicotómica; dicho de otro modo, el valor observado tiene o carece de la condición especificada (Cameron y Trivedi, 2010). De tal modo, para este estudio, la variable dependiente es asignada con los valores siguientes:

$$
y=\left\{\begin{array}{l}
1 \text { entorno propicio } \\
0 \text { entorno no propicio }
\end{array}\right.
$$

Es así como el valor 1 corresponde al entorno que es idóneo para que se desarrolle una empresa social y el valor 0 indica lo contrario. En primer lugar, para lograr la clasificación descrita, se contabilizaron las OSC por estado y se agruparon por entidad federativa con base en el estatus que presentaban a la fecha, es decir, activas o inactivas; esta información se puede observar en la tabla 2.

En segundo lugar, se utilizó un valor relativizado que proporcionó una mayor flexibilidad y facilidad sobre la categorización de la variable, el cual consistió en obtener la relación de empresas sociales activas respecto a las inactivas para cada entidad federativa. Por último, dicho valor se categoriza en cuartiles con el propósito de agrupar a los estados en estos rangos estadísticos y permitir una operacionalización más adecuada (Levie y Hart, 2011).

TABLA 2

Empresas sociales por entidad federativa

\begin{tabular}{lrrrlrrr}
\hline Entidad Federativa & Activas & Inactivas & Cuartil & Entidad federativa & Activas & Inactivas & Cuartil \\
\hline Aguascalientes & 204 & 198 & 1 & Morelos & 546 & 411 & 2 \\
Baja California & 727 & 572 & 2 & Nayarit & 205 & 184 & 1 \\
Baja California Sur & 165 & 94 & 4 & Nuevo León & 365 & 367 & 1 \\
Campeche & 126 & 86 & 3 & Oaxaca & 1656 & 937 & 4 \\
Chiapas & 1028 & 660 & 3 & Puebla & 873 & 510 & 4 \\
Chihuahua & 588 & 490 & 2 & Querétaro & 353 & 223 & 4 \\
Coahuila & 341 & 332 & 1 & Quintana Roo & 242 & 221 & 1 \\
Colima & 187 & 134 & 3 & San Luis Potosí & 255 & 182 & 3 \\
Ciudad de México & 4776 & 2710 & 4 & Sinaloa & 345 & 197 & 4 \\
Durango & 656 & 448 & 3 & Sonora & 444 & 247 & 4 \\
Estado de México & 1967 & 1363 & 3 & Tabasco & 285 & 254 & 1 \\
Guanajuato & 552 & 481 & 2 & Tamaulipas & 256 & 202 & 2 \\
Guerrero & 517 & 339 & 3 & Tlaxcala & 224 & 182 & 2 \\
Hidalgo & 504 & 324 & 3 & Veracruz & 1369 & 1243 & 1 \\
Jalisco & 788 & 698 & 2 & Yucatán & 436 & 180 & 1 \\
Michoacán & 836 & 754 & 1 & Zacatecas & 248 & 194 & 2 \\
\hline
\end{tabular}

Nota: elaboración propia a partir de datos recuperados de INDESOL (2018b).

Por lo tanto, se considera que las entidades federativas categorizadas en los cuartiles inferiores ( 1 y 2$)$ pertenecen a entornos que no son propicios para desarrollar empresas sociales, mientras que los cuartiles superiores $(3$ y 4$)$ dictaminan lo contrario. 


\subsection{Definición de variables independientes}

A manera de resumen, en la tabla 3 se presentan la operacionalización de las variables que intervienen en el estudio, la cual contiene información sobre los componentes del indicador y la fuente de donde se obtuvieron los datos.

TABLA 3

Descripción de las variables independientes

\begin{tabular}{|c|c|c|c|}
\hline Variable & Indicador & Descripción & Fuente \\
\hline $\begin{array}{l}\text { Ingreso } \\
\text { económico }\end{array}$ & $\begin{array}{l}\text { Logaritmo natural del ingreso per cápita a nivel } \\
\text { estado }\end{array}$ & $\begin{array}{l}\text { Índice que representa la capa- } \\
\text { cidad de ingreso per cápita y } \\
\text { de poder adquisitivo }\end{array}$ & $\operatorname{GDL}(2017)^{[3]}$ \\
\hline $\begin{array}{l}\text { Acceso a la } \\
\text { educación }\end{array}$ & $\begin{array}{l}\text { Es el resultado de la multiplicación del promedio } \\
\text { de años de escolaridad de adultos con edades de } \\
25 \text { años por el producto de la división del pro- } \\
\text { medio esperado de años de escolaridad entre el } \\
\text { promedio de años de escolaridad }\end{array}$ & $\begin{array}{l}\text { Representa la cantidad de } \\
\text { individuos que tienen acceso } \\
\text { a la educación }\end{array}$ & GDL (2017) $)^{[4]}$ \\
\hline $\begin{array}{l}\text { Acceso a la } \\
\text { salud }\end{array}$ & $\begin{array}{l}\text { Indica la expectativa de vida al nacer; es decir, } \\
\text { la cantidad de años que vivirían si estuviesen } \\
\text { sujetos a los riesgos de mortalidad existentes en } \\
\text { la población al momento de su nacimiento. }\end{array}$ & $\begin{array}{l}\text { Representa el porcentaje de } \\
\text { individuos que obtiene servi- } \\
\text { cios básicos de salud. }\end{array}$ & $\operatorname{GDL}(2017)^{[5]}$ \\
\hline Migración & $\begin{array}{l}\text { Indicador compuesto por cuatro categorías del } \\
\text { fenómeno de migración México-Estado Unidos. } \\
\text { Recolecta información de ingresos provenientes } \\
\text { del extranjero, emigrantes, migrantes circulares } \\
\text { y migrantes de retorno }\end{array}$ & $\begin{array}{l}\text { Representa la intensidad mi- } \\
\text { gratoria presente por entidad } \\
\text { federativa }\end{array}$ & CONAPO $(2010)^{[6]}$ \\
\hline Inseguridad & $\begin{array}{l}\text { Indicador que contempla cuatro aspectos relacio- } \\
\text { nados con seguridad: sensación de inseguridad } \\
\text { por miedo a un delito, ser testigo de actividades } \\
\text { delictivas, prevención y protección y cohesión } \\
\text { social. }\end{array}$ & $\begin{array}{l}\text { Percepción de la población } \\
\text { sobre la inseguridad de cada } \\
\text { entidad federativa }\end{array}$ & INEGI (2017) $)^{[7]}$ \\
\hline $\begin{array}{l}\text { Facilidad de } \\
\text { emprender }\end{array}$ & $\begin{array}{l}\text { Índice que aglomera datos sobre } 11 \text { áreas de regu- } \\
\text { laciones que tienen impacto en la creación de un } \\
\text { negocio. Entre estas áreas se encuentran: permisos } \\
\text { de construcción, acceso a créditos bancarios, tasas } \\
\text { de impuestos, regulaciones en contratos, entre } \\
\text { otros. Permite analizar los resultados económi- } \\
\text { cos y detectar que regulaciones han mejorado el } \\
\text { entorno de creación de empresas }\end{array}$ & $\begin{array}{l}\text { Representa la facilidad de } \\
\text { iniciar un negocio }\end{array}$ & World Bank (2019) ${ }^{[8]}$ \\
\hline
\end{tabular}

Nota: las variables han sido recolectadas del Global Data Lab (GDL), del Instituto Nacional de Estadística y Geografía (INEGI), el Consejo Nacional de Población (CONAPO) y el Banco Mundial-Doing Busines (World Bank).

Una vez establecidas las métricas de las variables, se procede a utilizar la técnica de regresión logística, la cual ha tenido utilización en estudios previos relacionados con el emprendimiento social (Estrin et al., 2013; Urbano et al., 2017, 2014). Además, al emplearlo se cumple con la demanda solicitada por la literatura sobre la elaboración de estudios que hagan uso de metodologías de tipo cuantitativas (Kachlami et al., 2018; Pathak y Muralidharan, 2018). En consecuencia, el estudio presentado permitirá determinar los factores que contribuyen a la existencia de empresas sociales en México.

Con base en lo relatado, se plantea el siguiente modelo econométrico:

$$
\operatorname{Pr}\left(\text { entorno propicio } 1 \mid x_{1} x_{2} x_{3} x_{4} x_{5} x_{6}\right)=\frac{1}{1+e\left(-\beta_{0}-\beta_{1} \chi_{1} \beta_{2} \chi_{2} \beta_{3} \chi_{3} \beta_{4} \chi_{4} \beta_{5} \chi_{5} \beta_{6} \chi_{6}+\epsilon\right)}
$$


En donde:

$\operatorname{Pr}\left(\right.$ entorno propicio $\left.1 \mid x_{1} x_{2} x_{3} x_{4} x_{5} x_{6}\right)$ es la probabilidad de que la variable dependiente tome el valor 1 en presencia de las covariables $x_{1} x_{2} x_{3} x_{4} x_{5} x_{6}$

$\beta_{0}$ es la constante del modelo.

$\beta_{1}$ es el coeficiente de la covariable del ingreso económico

$\beta_{2}$ es el coeficiente de la covariable del acceso a la educación.

$\beta_{3}$ es el coeficiente de la covariable del acceso a la salud.

$\beta_{4}$ es el coeficiente de la covariable de migración.

$\beta_{5}$ es el coeficiente de la covariable de percepción de inseguridad.

$\beta_{6}$ es el coeficiente de la covariable de la facilidad de emprender.

$\epsilon$ es el posible error de predicción para cada covariable.

$e$ representa la constante de Euler $(2,718)$.

\section{Resultados}

Para la predicción de ser un entorno propicio o no propicio para la probabilidad de existencia de empresas sociales, se utilizó una regresión logística categorizando la variable dependiente en empresas sociales activas y empresas sociales inactivas. El modelo logra efectuar una estimación correcta total de $78.1 \%\left(x^{2}=21.13, p<0.01\right)$, siendo los predictores de la ecuación el ingreso económico $(p=0.219)$, acceso a la educación $(p=0.031)$, acceso a la salud ( $p=0.421)$, migración $(p=0.268)$, inseguridad $(p=0.033)$ y la facilidad de emprender $(p=0.047)$.

En razón de conocer la contribución de cada variable al poder predictivo del modelo, se ejecutó la técnica mediante pasos sucesivos; en otras palabras, se introdujeron las variables en distintos bloques, las cuales permitieron observar el cambio en las predicciones acertadas al adicionar cada variable de manera individual. Lo anterior da como resultado que la variable ingreso económico contribuye en 3.2\%; acceso la educación, 6.2\%; acceso a la salud, 15.6\%; referente a la migración su contribución es marginal, es decir, no existe una mejora notable; la variable inseguridad adiciona $3.1 \%$ y la facilidad de emprender añade $6.2 \%$.

La tabla 4 presenta el resumen del modelo LOGIT (Pr entorno propicio $\left.=1 \mid x_{1} x_{2} x_{3} x_{4} x_{5} x_{6}\right)$ con los valores individuales de cada variable, lo que implica que las entidades federativas con un mayor acceso a la educación, y que proporcionan mayores facilidades para emprender, incrementan la posibilidad de que existan empresas sociales. Los resultados permiten aceptar las hipótesis $H_{1}, H_{2}, H_{3}$ y $H_{6}$, mientras rechaza $H_{4}$ y $H_{5}$.

TABLA 4

Resumen del modelo

\begin{tabular}{|lccc}
\hline & Odds Ratio & Std. Err. & Sig \\
\hline Constante & $9.36 \mathrm{e}+23$ & $4.20 \mathrm{e}+25$ & 0.219 \\
Ingreso económico & $5.23 \mathrm{e}-60$ & $2.92 \mathrm{e}-58$ & 0.015 \\
Acceso a la educación & $7.24 \mathrm{e}+36$ & $2.85 \mathrm{e}+38$ & 0.031 \\
Acceso a la salud & $1.05 \mathrm{e}-18$ & $5.39 \mathrm{e}-19$ & 0.421 \\
Migración & 1.94604 & 1.168728 & 0.268 \\
Inseguridad & 0.8998813 & 0.0445416 & 0.033 \\
Facilidad de emprender & 1.494787 & 0.3027266 & 0.047 \\
Predicciones correctas & $78.1 \%$ & & \\
Número de observaciones & 32 & & \\
\multicolumn{2}{c}{ Pseudo $R^{2}=0.4764 ; R^{2} \mathrm{CS}=0.483 ; R^{2} \mathrm{~N}=0.644 ; x^{2}=21.13 ; p=0.0017$} \\
\hline
\end{tabular}

Nota: elaboracion propia a partir de resultados obtenidos.

Donde: $R^{2} \mathrm{CS}=\mathrm{R}$ cuadrado de Cox y Snell; $R^{2} \mathrm{~N}=\mathrm{R}$ cuadrado de Nagelkerke. 
Siguiendo con el análisis individual de cada hipótesis, la hipótesis $H_{1}$ que gira en torno al ingreso económico indica una relación positiva y significativa al tener un $p$ valor de 0.015 ; sin embargo, el valor de su Odds Ratio es demasiado bajo, lo que indica una variación casi inexistente. Asimismo, $\mathrm{H}_{2}$, correspondiente al acceso a la educación, resulta tener relación con la existencia de empresas sociales, la cual es significativa, con un $p$ valor de 0.031, cuyo valor de su Odds Ratio es positivo, lo que indica que un incremento en ella ocasionará un aumento en la probabilidad de existencia de empresas sociales.

Por su parte, la hipótesis $\mathrm{H}_{3}$, correspondiente al acceso a la salud, mantiene una relación positiva, pero carece de significatividad dado que su $p$ valor acepta la hipótesis nula, por lo cual se rechaza. Respecto a la hipótesis $H_{4}$, migración, la relación es inversa y no demuestra significatividad al aceptar, de igual manera, la hipótesis nula. De igual modo, $\mathrm{H}_{5}$, hipótesis correspondiente a la inseguridad, es rechazada al demostrar una relación contraria a la planteada, pese ser significativa.

Finalmente, la hipótesis de la facilidad de emprender se acepta, puesto que obtiene una asociación positiva y significativa, dado que su $p$ valor es de 0.047 . En este sentido, se puede interpretar que la educación y la facilidad de emprender proporcionan una mayor probabilidad en la existencia de empresas sociales, es decir, si la educación y la facilidad se incrementan, la probabilidad de que existan empresas sociales aumenta, lo que demuestra coincidencias con lo planteado en la revisión de la literatura.

\section{Discusión}

Con base en los resultados anteriores, se permite constatar la relación entre la existencia de empresas sociales y los factores del entorno presentes en México al lograr que cumpla con el objetivo trazado. Entre dichos resultados, destaca una vinculación significativa con el ingreso económico, el acceso a la educación y la facilidad de emprender.

En primer lugar, el ingreso económico tiene la relación y efecto esperado, además de poseer significancia; sin embargo, esta asociación debe de tomarse con cautela, a consecuencia de una nula variación en sus valores de ratio. Bajo este panorama, se encuentra evidencia empírica que soporta lo aludido por Pathak y Muralidharan (2018) acerca de la desigualdad en el ingreso económico de la población, escenario que genera que más individuos tengan la intención de emprender socialmente.

En segundo lugar, el acceso a la educación apoya las aportaciones de Estrin et al. (2013) y de Kachlami et al. (2018). Este resultado toma mayor relevancia, puesto que el primer estudio se desarrolla a nivel macro al evaluar múltiples países, mientras que el segundo es ubicado en Suecia, país con alto índice de desarrollo, y se suma a la correlación encontrada en el contexto español por Urbano et al. (2017) sobre la educación y el emprendedor social.

Asimismo, se hace mención de que las variables de acceso a la salud, migración e inseguridad no poseen una relación significativa con la existencia de empresas sociales, lo que obliga a que las hipótesis correspondientes a estas variables sean rechazadas. No obstante, el acceso a la salud demuestra una relación como la que plantea la literatura (Roy et al., 2014; Spear et al., 2009); un caso contrario es el resultado de los factores de migración e inseguridad.

Lo anterior presume ser resultado a los diferentes esfuerzos que ha realizado el gobierno mexicano para intentar disminuir las problemáticas que afligen a su población. Dentro de estos esfuerzos, se encuentran iniciativas y programas operados por el sector público que versan sobre estos factores. Ejemplo de ello, el programa Seguro Popular que tuvo por objetivo cubrir a la población que se encontraba en condición vulnerable en el apartado de salud al carecer de prestaciones médicas (Felix-Gonzalez, 2011).

Respecto a la migración, el resultado es contrario al planteado a priori en la revisión de la literatura, pero es concordante con lo marcado por Kachlami et al. (2018), quienes indican que el emprendimiento social ocurre en áreas que se encuentran sumamente urbanizadas. En este sentido, Saiz-Álvarez (2018), en su estudio descriptivo sobre Jalisco, indica que si bien el emprendimiento social ha podido disminuir la tasa de migración, dicha variación ha sido mínima, y deriva en que la población continúe migrando hacia la búsqueda de mejores oportunidades de vida. 
Empero, la variable de seguridad resulta ser interesante, dado que es una problemática que no ha podido ser solventada por México (Saiz-Álvarez, 2018) y que debería ser abordada por la creación de modelos de negocio del tipo social (Hlady-Rispal y Servantie, 2017). Sin embargo, ocurre lo contrario encontrándose respuestas similares a las planteadas por Hoogendoorn (2016), quien sugiere que un entorno de alta seguridad es el idóneo para crear empresas sociales. De esta manera, la aceptación de la hipótesis nula refuerza los planteamientos clásicos provenientes del emprendimiento tradicional, los cuales sugieren que los emprendedores optan por seleccionar entornos seguros para ubicar nuevas iniciativas de negocio (Rosenthal y Ross, 2010).

En este sentido, en Hidalgo y Oaxaca la percepción de la inseguridad resulta ser baja en comparación con las demás entidades federativas (INEGI, 2017). En Hidalgo se han detectado empresas sociales mediante la evaluación de resultados de programas de incubación (Amador, Cadena y Castro, 2015), mientras que en Oaxaca se han localizado e investigado empresas sociales a través de estudios cualitativos (Fernández et al., 2012; Wulleman y Hudon, 2016); en consecuencia, se obtiene congruencia entre las ubicaciones donde se han efectuado estos estudios y la probabilidad de existencia de empresas sociales en entornos seguros.

Por último, la variable de facilidad de iniciar un negocio resulta ser significativa. Dicha significancia, concuerda con lo encontrado por Urbano et al. (2017), quienes manifiestan la importancia que reside en la capacidad de acceder, por parte del emprendedor social, a diversas fuentes financiaras, situación que le brinda la solvencia necesaria para operar durante los primeros años de vida de la organización. A su vez, denota la necesidad de impulsar a este tipo de empresas por parte del sector público mexicano mediante la creación de leyes y programas que continúen con el apoyo a los emprendedores sociales (Felix-Gonzalez, 2011; Wulleman y Hudon, 2016).

\section{Conclusiones y PROSPECTIVA}

El objetivo de esta investigación fue la determinación de los factores del entorno que contribuyen en la existencia de empresas sociales en México, específicamente en la probabilidad de que suceda dicho fenómeno. Para lograrlo, se utilizó información proveniente de diversas fuentes secundarias que representan variables internas de las entidades federativas del país y se revela que los factores son similares a los contextualizados en investigaciones efectuadas en países altamente desarrollados.

Considerando lo anterior, es oportuno manifestar que este artículo es una de las primeras contribuciones empíricas en América Latina y permite reforzar la literatura existente sobre el emprendimiento social (Félix González et al., 2017; Hlady-Rispal y Servantie, 2017; Saiz-Álvarez, 2018) al abordar un tema necesitado de conocimiento como es lo concerniente con la empresa social. Esta situación permitirá acrecentar el interés por comprender el funcionamiento de este tipo de organización desde diferentes puntos de vista como el académico, el empresarial o el gubernamental, lo que trae conciencia de la importancia que tiene dicho modelo empresarial en la vida económica y social de las personas (Spear et al., 2009; Urbano et al., 2010).

No obstante, el estudio presenta limitaciones a consecuencia de que se emplea una definición proporcionada por una institución del sector público en lugar de una identificada por la literatura; esta situación imposibilita lograr la descripción, análisis y medición del impacto que tiene la empresa social al establecer únicamente los factores que incrementan la posibilidad de que dichos modelos de negocio existan.

Por último, y con la finalidad de abordar las limitaciones del estudio, se bosquejan futuras líneas de investigación que permitan fortalecer el tópico del emprendimiento social. Referente a eso, se encuentra el caso de efectuar estudios empíricos en países de América Latina, los cuales resguardan características similares al entorno mexicano; por ejemplo, indagar sobre la intención de emprender socialmente, así como de anexar factores exclusivos de cada país. Además, deberán versar sobre las características que tienen las empresas sociales, así como los elementos que las llevan al éxito o al fracaso. Para ello, se deben efectuar investigaciones empíricas que permitan generalizar resultados a vías de compararlas en el futuro. 


\section{Agradecimientos}

Se agradecen los comentarios de los árbitros de la revista que mejoraron sustancialmente el contenido del artículo.

\section{REFERENCIAS}

Amador, E. M. P., Cadena, M. G., \& Castro, J. A. V. (2015). Incubadoras sociales en las universidades como promotoras de innovación y empreendimiento social para el desarrollo de las regiones: el caso del Estado de Hidalgo, México. Revista Brasileira de Planejamento e Desenvolvimento, 4(1), 128-148. https://doi. org/10.3895/rbpd.v4n1.3598.

Aparicio, S., Urbano, D., \& Audretsch, D. (2016). Institutional factors, opportunity entrepreneurship and economic growth: Panel data evidence. Technological Forecasting and Social Change, 102, 45-61. https:// doi.org/10.1016/j.techfore.2015.04.006

Austin, J., Stevenson, H., \& Wei-Skillern, J. (2006). Social and commercial entrepreneurship: Same, different, or both? Entrepreneurship Theory and Practice, 30(1), 1-22. https://doi.org/10.1111/j.15406520.2006.00107.x

Bacq, S., \& Janssen, F. (2011). The multiple faces of social entrepreneurship: A review of definitional issues based on geographical and thematic criteria. Entrepreneurship \& Regional Development, 23(5-6), 373403. https://doi.org/10.1080/08985626.2011.577242

Braga, J. C., Proença, T., \& Ferreira, M. R. (2014). Motivations for social entrepreneurship-Evidences from Portugal. Tékhne, 12, 11-21. https://doi.org/10.1016/j.tekhne.2015.01.002

Cameron, A., \& Trivedi, P. (2010). Microeconometrics using stata (Revised). USA: Stata Press.

Castaño, M.-S., Méndez, M.-T., \& Galindo, M.-Á. (2015). The effect of social, cultural, and economic factors on entrepreneurship. Journal of Business Research, 68(7), 1496-1500. https://doi.org/10.1016/j. jbusres.2015.01.040

CONAPO. (2010). Índices de intensidad migratoria México-Estados Unidos 2010. Consejo Nacional de Población. Disponible en http://www.conapo.gob.mx/swb/CONAPO/Indices_de_intensidad_migratoria_Mexico-Estados_Unidos_2010

Dwivedi, A., \& Weerawardena, J. (2018). Conceptualizing and operationalizing the social entrepreneurship construct. Journal of Business Research, 86, 32-40. https://doi.org/10.1016/j.jbusres.2018.01.053

Estrin, S., Mickiewicz, T., \& Stephan, U. (2013). Entrepreneurship, social capital, and institutions: Social and commercial entrepreneurship across nations. Entrepreneurship Theory and Practice, 37(3), 479-504. https://doi.org/10.1111/etap.12019

Felix Gonzalez, M., Husted, B. W., \& Aigner, D. J. (2017). Opportunity discovery and creation in social entrepreneurship: An exploratory study in Mexico. Journal of Business Research, 81, 212-220. https:// doi.org/10.1016/j.jbusres.2016.10.032

Felix-Gonzalez, M. (2011). Social entrepreneurship in Mexico: A conceptual analysis. International Journal of Business Competition and Growth, 1(4), 314-331. https://doi.org/10.1504/IJBCG.2011.038696

Fernández, M. J., Castillejos, B., \& Ramírez, J. A. (2012). Empresas sociales y ecoturismo en Bahías de Huatulco, México. Diagnóstico de la gestión empresarial. Estudios y Perspectivas en Turismo, 21(1), 203-224.

García-Macias, M. A., Zerón-Félix, M., \& Sánchez-Tovar, Y. (2018). Factores de entorno determinantes del emprendimiento en México. Entramado, 14(1), 88-103. https://doi.org/10.18041//entramado.2018v14n1.27121 
Gartner, W. (1985). A conceptual framework for describing the phenomenon of new venture creation. The Academy of Management Review, 10(4), 696-706. https://doi.org/10.2307/258039

GDL. (2017). Global Data Lab-Subnational Human Development Index. Retrieved from https://globaldatalab.org/shdi/

Hlady-Rispal, M., \& Servantie, V. (2017). Business models impacting social change in violent and poverty-stricken neighbourhoods: A case study in Colombia. International Small Business Journal, 35(4), 427-448. https://doi.org/10.1177/0266242615622674

Hoogendoorn, B. (2016). The prevalence and determinants of social entrepreneurship at the macro level. Journal of Small Business Management, 54, 278-296. https://doi.org/10.1111/jsbm.12301

INDESOL. (2018a). Instituto Nacional de Desarrollo Social-Registro Federal de las OSC. Disponible en http://www.gob.mx/indesol/acciones-y-programas/registro-federal-de-las-osc

INDESOL. (2018b). Directorios de organizaciones de la sociedad civil inscritas en el Registro Federal de las OSC 2018. Disponible en https://datos.gob.mx/busca/dataset/registro-federal-de-las-organizaciones-de-la-sociedad-civil/resource/0d87a930-fde4-4e1b-9e96-352722081538

INEGI. (2017). Instituto Nacional de Estadística y Geografía-Encuesta Nacional de victimización y percepción sobre seguridad pública. Disponible en http://www.beta.inegi.org.mx/programas/ envipe/2017/

Inter-American Development Bank. (2016). Study of social entrepreneurship and innovation ecosystems in the Latin American pacific alliance countries. Country analys Mexico (pp. 1-18). Fundación Ecología y Desarrollo.

Kachlami, H., Yazdanfar, D., \& Öhman, P. (2018). Regional demand and supply factors of social entrepreneurship. International Journal of Entrepreneurial Behavior \& Research, 24(3), 714-733. https://doi. org/10.1108/IJEBR-09-2016-0292

Levie, J., \& Hart, M. (2011). Business and social entrepreneurs in the UK: Gender, context and commitment. International Journal of Gender and Entrepreneurship, 3(3), 200-217. https://doi. org/10.1108/17566261111169304

Littlewood, D., \& Holt, D. (2018). Social entrepreneurship in South Africa: Exploring the influence of environment. Business \& Society, 57(3), 525-561. https://doi.org/10.1177/0007650315613293

Mair, J., \& Martí, I. (2006). Social entrepreneurship research: A source of explanation, prediction, and delight. Journal of World Business, 41(1), 36-44. https://doi.org/10.1016/j.jwb.2005.09.002

North, D. (1991). Institutions. The Journal of Economic Perspectives, 5(1), 97-112.

Pathak, S., \& Muralidharan, E. (2018). Economic inequality and social entrepreneurship. Business \& Society, 57(6), 1150-1190. https://doi.org/10.1177/0007650317696069

Rosenthal, S. S., \& Ross, A. (2010). Violent crime, entrepreneurship, and cities. Journal of Urban Economics, 67(1), 135-149. https://doi.org/10.1016/j.jue.2009.09.001

Roy, M. J., Donaldson, C., Baker, R., \& Kerr, S. (2014). The potential of social enterprise to enhance health and well-being: A model and systematic review. Social Science \& Medicine, 123, 182-193. https://doi. org/10.1016/j.socscimed.2014.07.031

Saiz-Álvarez, J. M. (2018). Turismo sostenible y emprendimiento social. El Pueblo Mágico de Tequila, México. Retos, 8(15), 51-67. https://doi.org/10.17163/ret.n15.2018.04

Seelos, C., \& Mair, J. (2005). Social entrepreneurship: Creating new business models to serve the poor. Business Horizons, 48(3), 241-246. https://doi.org/10.1016/j.bushor.2004.11.006 
Smits, J., \& Permanyer, I. (2019). The subnational human development database. Scientific Data, 6, 190038. Retrieved from https://www.nature.com/articles/sdata201938

Son, H., Lee, J., \& Chung, Y. (2017). Value creation mechanism of social enterprises in manufacturing industry: Empirical evidence from Korea. Sustainability, 10(46), 1-24. https://doi.org/10.3390/ su10010046

Spear, R., Cornforth, C., \& Aiken, M. (2009). The governance challenges of social enterprises: Evidence from a Uk empirical study. Annals of Public and Cooperative Economics, 80(2), 247-273. https://doi.org/10.1111/ j.1467-8292.2009.00386.x

Urbano, D., Ferri, E., Alvarez, C., \& Noguera, M. (2017). Social entrepreneurship and institutional conditions: An empirical analysis in Spain. Social Entrepreneurship in Non-Profit and Profit Sectors, 36, 53-64. https:// doi.org/10.1007/978-3-319-50850-4_4

Urbano, D., Ferri, E., \& Noguera, M. (2014). Female social entrepreneurship and socio-cultural context: An international analysis. Revista de Estudios Empresariales, 1(2), 26-40.

Urbano, D., Toledano, N., \& Soriano, D. R. (2010). Analyzing social entrepreneurship from an institutional perspective: Evidence from Spain. Journal of Social Entrepreneurship, 1(1), 54-69. https://doi. org/10.1080/19420670903442061

World Bank. (2019). World Bank-Doing Business. Retrieved from http://espanol.doingbusiness.org/es/doingbusiness

Wulleman, M., \& Hudon, M. (2016). Models of social entrepreneurship: Empirical evidence from Mexico. Journal of Social Entrepreneurship, 7(2), 162-188. Retrieved from https://doi.org/10.1080/19420676.2 015.1057207

Zahra, S. A., Rawhouser, H. N., Bhawe, N., Neubaum, D. O., \& Hayton, J. C. (2008). Globalization of social entrepreneurship opportunities. Strategic Entrepreneurship Journal, 2(2), 117-131. https://doi.org/10.1002/ sej. 43

\section{Notas}

[1] En México las instituciones encargadas de administrar la forma en que los emprendimientos se realizan son INDESOL y SEDESOL(Inter-American Development Bank, 2016). Cabe aclarar que en el año 2018 SEDESOL cambió de nombre a Secretaría de Bienestar.

[2] Disponible en https://www.gob.mx/indesol/acciones-y-programas/registro-federal-de-las-osc.

[3] Para profundizar en el cálculo del indicador, véase Smits y Permanyer (2019).

[4] Para profundizar en el cálculo del indicador, véase Smits y Permanyer (2019).

[5] Para profundizar en el cálculo del indicador, véase Smits y Permanyer (2019).

[6] Para profundizar en el cálculo del indicador, véase CONAPO (2010).

[7] Para profundizar en el cálculo del indicador, véase INEGI (2017).

[8] Para profundizar en el cálculo del indicador, véase World Bank (2019).

\section{BY-NC-ND}

